\title{
Permanência do Cateter de Hickman em Pacientes Submetidos a Transplante de Células-Tronco Hematopoéticas Alogênico: Estudo Retrospectivo
}

\author{
Permanence of Hickman Catheter in Patients Undergoing Allogeneic \\ Hematopoietic Stem Cell Transplantation: Retrospective Study \\ Permanencia del Catéter Hickman en Pacientes Sometidos a Trasplante Alogénico \\ de Células Madre Hematopoyéticas: Estudio Retrospectivo
}

\author{
Juliane Zagatti Alves Pereira ; Fernanda Titareli Merizio Martins Braga ${ }^{2}$; Lívia Maria Garbin ${ }^{3}$; Lais Carvalho Castanho ${ }^{4}$; Renata Cristina de Campos \\ Pereira Silveira ${ }^{5}$
}

\section{Resumo}

Introduçáo: $\mathrm{O}$ uso do cateter de Hickman é fundamental para viabilizar terapêuticas complexas, no entanto, sua permanência segura requer o conhecimento dos profissionais que o manuseiam sobre as principais complicaçóes que levam à sua retirada. Objetivo: Analisar a permanência do cateter de Hickman em pacientes submetidos ao transplante de células-tronco hematopoéticas alogênico. Método: Estudo descritivo do tipo transversal, com coleta de dados retrospectiva. Foram identificados 73 prontuários de pacientes no período de interesse do estudo, dos quais três foram excluídos por possuírem informaçôes incompletas. Os dados foram analisados por meio de estatística descritiva. Resultados: Foram implantados 78 cateteres em 70 pacientes, sendo o tempo médio de permanência dos dispositivos de 41 dias ( $\mathrm{DP}=26,78)$. A média do tempo transcorrido entre o implante do cateter e o início do regime de condicionamento foi quatro dias $(\mathrm{DP}=4,6)$. Em $26(33,4 \%)$ casos, a indicação de retirada do dispositivo ocorreu por suspeita de infecçóes relacionadas ao cateter e, em outros $24(30,8 \%)$ casos, a retirada ocorreu após seguimento ambulatorial, sem complicação documentada no prontuário. Conclusão: $\mathrm{O}$ cateter de Hickman geralmente causa complicaçóes, entre elas, a infecção, o que leva a um curto tempo de permanência do dispositivo in situ, contrariando sua proposta de longa permanência; além de muitas vezes ser retirado sem registro do critério adotado, antes do término da terapia. Esta pesquisa contribui com o enfermeiro da prática clínica ao identificar os principais motivos de retirada do cateter e ao fornecer dados que podem subsidiar propostas de intervençôes para minimizá-los.

Palavras-chave: Cateterismo Venoso Central-enfermagem; Cuidados de Enfermagem; Transplante de Medula Ósseaenfermagem; Infecçôes Relacionadas a Cateter-enfermagem; Transplante de Células-Tronco Hematopoéticas-enfermagem

\footnotetext{
Este estudo teve apoio financeiro da Fundaçáo de Amparo e Ensino à Pesquisa do Estado de São Paulo (FAPESP), processo no $2010 / 12704-4$.

${ }^{1}$ Enfermeira. Ex-bolsista de Iniciação Científica da FAPESP, Escola de Enfermagem de Ribeirão Preto da Universidade de São Paulo (EERP-USP). Ribeirão Preto (SP), Brasil. E-mail: ju_zagatti@hotmail.com.

${ }^{2}$ Enfermeira. Doutora em Ciências. Enfermeira da EERP-USP. Ribeirão Preto (SP), Brasil. E-mail: titareli@eerp.usp.br.

${ }^{3}$ Enfermeira. Doutoranda em Ciências. Enfermeira da EERP-USP. Ribeirão Preto (SP), Brasil. E-mail: liviagarbin@usp.br.

${ }^{4}$ Enfermeira. Especialista em Enfermagem Oncológica e Tratamento Antineoplásico. Mestranda em Ciências. EERP-USP. Ribeirão Preto (SP), Brasil.

E-mail: lais.castanho@gmail.com.

${ }^{5}$ Enfermeira. Doutora em Ciências. Professor Doutor da EERP-USP. Ribeirão Preto (SP), Brasil. E-mail: recris@eerp.usp.br.

Endereço para correspondência: Renata Cristina de Campos Pereira Silveira. Av. Bandeirantes, 3.900. Monte Alegre. Ribeirão Preto (SP), Brasil. CEP: $14040-902$.
} 


\section{INTRODUÇÃO}

O transplante de células-tronco hematopoéticas (TCTH) consiste na infusão de células-tronco hematopoéticas (CTH), sendo que, entre as condições nas quais essa terapêutica pode ser empregada, estão as doenças malignas, as doenças genéticas e as doenças autoimunes. Os transplantes são denominados singênicos quando o doador das CTH é um gêmeo univitelino, alogênicos nos casos em que estas provêm de outro doador, e autólogo quando são procedentes do próprio paciente ${ }^{1}$.

Apesar dos benefícios na sobrevida e/ou cura da doença de base, esse procedimento é considerado de risco devido às complicaçôes que podem surgir, cuja frequência depende do tipo de transplante efetuado, bem como da idade, doença de base, estádio da doença e da condição clínica do paciente ${ }^{2}$.

Anterior à infusão das $\mathrm{CTH}$, o paciente é submetido a altas doses de quimioterapia associadas ou não à irradiação corporal total para erradicar a doença de base, suprimir a medula óssea e prevenir a rejeiçấo das células do doador no caso do transplante alogênico. Esse período é conhecido como regime de condicionamento, cuja intensidade está associada ao aumento da morbidade e mortalidade ${ }^{1}$.

Com o propósito de infundir as CTH pelo sistema venoso de maneira segura, aliado à necessidade de terapêutica endovenosa por tempo prolongado, requer-se a inserção de um cateter venoso central de longa permanência antes do início do regime de condicionamento ${ }^{3}$, sendo que o cateter de Hickman foi o pioneiro e tem sido utilizado até os dias atuais para essa terapêutica.

No entanto, ainda que esse dispositivo ofereça pontos positivos, sua presença pode ocasionar complicaçóes capazes de aumentar a morbidade entre os pacientes, as quais sáo classificadas como precoces (arritmia, mau posicionamento, punção arterial, embolia gasosa, pneumotórax, deiscência, hemorragia, e mais raramente danos a vasos e nervos) ou tardias (infecção, fratura e embolização do cateter, migração, mau funcionamento e trombose $)^{4}$. Entre elas, a infecção causada pela quebra da integridade cutânea do paciente ${ }^{5-6}$ está relacionada a um aumento significativo da mortalidade nos primeiros seis meses após o implante, além de levar ao aumento do período de internaçáa ${ }^{7}$.

Sabe-se que o momento do implante cirúrgico do cateter de Hickman, como primeira ação preparatória para o TCTH, é considerado como o de melhor condição clínica do paciente. Assim, se sua substituição posterior a esse momento for necessária, ocorrerá em condiçóes clínicas menos favoráveis e com maior morbidade ${ }^{8}$.

As condiçóes clínicas do paciente podem ser preditoras do aparecimento de infecçóes relacionadas ao cateter, assim como o quadro de neutropenia acima de 10 dias, associado ao uso do cateter para infusão de altas doses de quimioterápicos e de nutrição parenteral total (NPT) ${ }^{9}$.
Dessa forma, considera-se de extrema relevância que os enfermeiros e demais profissionais da saúde, responsáveis diariamente pelo manuseio do cateter venoso central, tenham o conhecimento acerca das principais complicaçóes que levam à retirada desse dispositivo, para que possam planejar e implementar açóes que garantam sua permanência e minimizem os riscos para os pacientes.

Diante do exposto, essa investigaçáo teve como objetivo principal: analisar a permanência do cateter de Hickman em pacientes submetidos ao TCTH alogênico, e específicos: desenvolver essa análise considerando o tempo de permanência do cateter in situ, o intervalo de tempo entre o procedimento cirúrgico e o início do regime de condicionamento, os motivos de retirada, e a ocorrência de complicaçōes tardias relacionadas ao cateter de Hickman.

\section{MÉTODO}

Trata-se de um estudo descritivo, transversal, retrospectivo, realizado em uma unidade de TCTH, de um hospital universitário, público e geral do interior do Estado de São Paulo.

Teve como populaçáo-alvo sujeitos adultos (idade igual ou superior a 18 anos), submetidos ao TCTH alogênico no período de 2006 a 2010, e que tiveram o cateter de Hickman implantado no centro cirúrgico. Assim, 73 pacientes atenderam aos critérios de inclusão; no entanto, três foram excluídos por apresentarem dados incompletos no prontuário. A coleta de dados foi realizada no primeiro semestre de 2011.

Para a coleta de dados, foi elaborado um instrumento contendo itens de identificaçáo do paciente, dados demográficos, clínicos e aspectos relacionados à permanência do cateter de Hickman. Foi feita validação de aparência e conteúdo por três juízes com experiência profissional na assistência a pacientes submetidos ao TCTH e realizadas sugestôes quanto à forma de apresentaçáo dos itens, sendo todas acatadas.

Em relaçáo ao intervalo de tempo transcorrido entre o dia de implante do cateter e o início do regime de condicionamento, foi considerado zero para os regimes de condicionamento que começaram no mesmo dia que o cateter foi implantado. A mesma contagem se aplicou quando o dispositivo foi retirado no mesmo dia do implante. A neutropenia severa foi considerada quando a contagem de neutrófilos era igual ou inferior a 500 células $/ \mathrm{mm}^{3}$ e sem tendência ao aumento. Já a enxertia dos neutrófilos foi considerada quando os valores laboratoriais indicavam contagem superior a 500 células $/ \mathrm{mm}^{3}$, por pelo menos três dias consecutivos. Para a classificação da infecção de corrente sanguínea, foram considerados os critérios preconizados pelos Centers for Disease Control and Prevention ${ }^{10}$.

O estudo foi aprovado pelo Comitê de Ética em Pesquisa (CEP) da Instituição na qual foi realizado 
(protocolo no 4909/2010) e, após o levantamento e disponibilização dos prontuários no Serviço de Arquivo Médico, o instrumento de coleta de dados foi preenchido.

Os dados coletados foram armazenados em uma planilha eletrônica do Microsoft Excel $2010^{\circ}$. Para garantir a fidedignidade da digitação, foi realizada a dupla alimentaçáo do banco de dados e, após correçáo, os dados foram transferidos para o programa Statistical Package for Social Science (SPSS), versão 17.0 para Windows, e então realizada sua análise descritiva. Para as variáveis numéricas, foram calculados os valores médios e seus respectivos desvios-padrão, e, para as variáveis qualitativas, a frequência absoluta e porcentagem.

\section{RESULTADOS}

A amostra foi composta por prontuários de 70 pacientes, sendo a maioria do sexo masculino $(57,1 \%)$. A idade variou de 18 a 64 anos, com média de 36 anos. A patologia de base mais frequente foi a leucemia mieloide aguda (LMA) (28,6\%), seguida pela anemia aplásica grave $(22,8 \%)$. A associação de fludarabina e bussulfano foi o principal regime de condicionamento utilizado $(22,8 \%)$ (Tabela 1).

O maior tempo para confirmação da enxertia dos neutrófilos foi de 22 dias, com mediana de 16 dias. Do total de pacientes, 94,3\% evoluíram para neutropenia severa, com duração média de 17 dias $(\mathrm{DP}=10,5)$. Do total da amostra, 43 sujeitos $(61,4 \%)$ apresentaram mucosite e a NPT foi utilizada em 36 indivíduos (51,4\%).

Tendo em vista que oito pacientes tiveram dois cateteres implantados, foram analisados os dados relacionados a 78 dispositivos. O procedimento cirúrgico para implante do cateter teve duração média de 52 minutos, sem intercorrências na maioria dos casos $(88,5 \%)$. Com relaçáo ao tempo de permanência do dispositivo in situ, a média foi de 41 dias, com mínimo de zero e máximo de 118 dias, sendo que houve três casos nos quais foi necessária a retirada do CVC antes de completar um dia de implante devido a mau posicionamento $(\mathrm{n}=2)$ e obstruçáo $(\mathrm{n}=1)$. A retirada foi indicada, na maioria das vezes $(96,2 \%)$, por médico residente da hematologia, sendo o ambulatório o local de retirada mais frequente (46,2\%); enquanto $39,7 \%$ ocorreram com o paciente ainda internado na enfermaria (Tabela 2).

Em 24 situações $(30,8 \%)$, a indicação de retirada ocorreu após o paciente iniciar o seguimento ambulatorial, porém sem complicação documentada; seguidos por 19 casos $(24,4 \%)$ nos quais a retirada foi indicada por suspeita de infecção da corrente sanguínea relacionada ao cateter venoso central (ICSR-CVC). As razóes para retirada dos demais 35 cateteres $(44,8 \%)$ foram variadas (Tabela 2$)$.

$\mathrm{O}$ intervalo transcorrido entre o implante do cateter de Hickman e o início do regime de condicionamento
Tabela 1. Caracterização dos pacientes submetidos ao transplante de células-tronco hematopoéticas alogênico em um hospital universitário $(\mathrm{N}=70)$, segundo as variáveis sociodemográficas e clínicas, Ribeirão Preto-SP, 2006-2010

\begin{tabular}{|c|c|c|}
\hline VARIÁVEIS & $\mathbf{n}$ & $\%$ \\
\hline \multicolumn{3}{|l|}{ Sexo } \\
\hline Masculino & 40 & 57,1 \\
\hline Feminino & 30 & 42,9 \\
\hline \multicolumn{3}{|l|}{ Idade (anos) } \\
\hline Média (DPI) & $36,7(12,4)$ & \\
\hline Mediana & 36,5 & \\
\hline Variação & $18-64$ & \\
\hline \multicolumn{3}{|l|}{ Patologia de base } \\
\hline Leucemia mieloide aguda & 20 & 28,6 \\
\hline Anemia aplásica grave & 16 & 22,8 \\
\hline Leucemia linfocítica aguda & 8 & 11,4 \\
\hline Síndrome mielodisplásica & 7 & 10 \\
\hline Leucemia mieloide crônica & 7 & 10 \\
\hline Anemia falciforme & 3 & 4,3 \\
\hline Mieloma múltiplo & 2 & 2,9 \\
\hline Outras" & 7 & 10 \\
\hline \multicolumn{3}{|c|}{ Regime de condicionamento"' } \\
\hline FLU BU & 16 & 22,8 \\
\hline TANDEM & 13 & 18,6 \\
\hline FLU BU ATG & 11 & 15,7 \\
\hline CY ATG & 6 & 8,6 \\
\hline BU CY & 4 & 5,7 \\
\hline CY TBI & 4 & 5,7 \\
\hline FLU CY TBI & 4 & 5,7 \\
\hline Outros $^{\mathrm{IV}}$ & 12 & 17,2 \\
\hline
\end{tabular}

'DP: Desvio-padrāo; "leucemia linfocítica crônica, linfoma não Hodgkin, leucemia bifenotípica aguda, trombastenia de Glanzmann, síndrome Blackfan-Diamond, síndrome MNGIE (Mitocondrial Neuro Gastro Intestinal Encephalopathy), meduloblastoma; "II FLU: fludarabina, BU: bussulfano, TANDEM: fludarabinacitarabina-mitoxantrone-bussulfano-ciclofosfamida-globulina antitimocitária, ATG: globulina antitimocitária, CY: ciclofosfamida, TBI: irradiação corporal

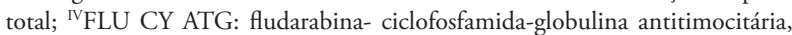
CY: ciclofosfamida, CY ATG: ciclofosfamida-globulina antitimocitária, FLU MEL: fludarabina e melfalano, FLU MEL ATG: fludarabina-melfalano-globulina antitimocitária, FLU CY: fludarabina e ciclofosfamida

foi em média de 3,9 dias, (mediana de três dias, variação de zero a 36 dias, $\mathrm{DP}=4,5$ ), sendo que o período inferior a quatro dias foi constatado em $60 \%$ dos casos $(n=42)$.

Dos 19 cateteres retirados por suspeita de ICSRCVC, nove $(47,4 \%)$ tiveram crescimento do mesmo microrganismo na hemocultura e na cultura da ponta do cateter, totalizando dez microrganismos isolados, dos quais, oito $(80 \%)$ eram gram-negativos e dois (20\%) eram gram-positivos. Ressalta-se que, em dois cateteres, a hemocultura foi positiva para dois tipos 
de microrganismos, entretanto, em um destes foi isolado somente um microrganismo na ponta. Os microrganismos mais frequentes foram $K$. pneumoniae e A. baumannii, correspondendo a $30 \%(\mathrm{n}=3)$ cada um. Em um caso, a cultura de ponta do cateter foi negativa e nos demais não foram encontrados nos prontuários registros dos resultados obtidos (Tabela 3).

Tabela 2. Dados relacionados ao procedimento cirúrgico de implante, tempo de permanência in situ e caracterização da retirada do cateter de Hickman (N=78), Ribeirão Preto-SP, 2006-2010

\begin{tabular}{|c|c|c|}
\hline VARIÁVEIS & $\mathbf{n}$ & $\%$ \\
\hline \multicolumn{3}{|l|}{ Duração do implante (minutos) } \\
\hline Média (DPII) & \multicolumn{2}{|c|}{$52,4(29,1)$} \\
\hline Mediana & \multicolumn{2}{|c|}{50} \\
\hline Variação & \multicolumn{2}{|c|}{$10-180$} \\
\hline \multicolumn{3}{|l|}{ Intercorrências no centro cirúrgico } \\
\hline Nenhuma & 69 & 88,5 \\
\hline Mau posicionamento & 3 & 3,8 \\
\hline Várias punções & 2 & 2,6 \\
\hline Outros' & 4 & 5,1 \\
\hline \multicolumn{3}{|c|}{ Tempo de permanência do cateter (dias) } \\
\hline Média (DPII) & \multicolumn{2}{|c|}{$41,4(26,7)$} \\
\hline Mediana & \multicolumn{2}{|c|}{36} \\
\hline Variação & \multicolumn{2}{|c|}{$0-118$} \\
\hline \multicolumn{3}{|l|}{ Profissional que indicou a retirada } \\
\hline Médico residente da hematologia & 75 & 96,2 \\
\hline Outros ${ }^{\prime \prime \prime}$ & 3 & 3,8 \\
\hline \multicolumn{3}{|l|}{ Local de retirada } \\
\hline Ambulatório & 36 & 46,2 \\
\hline Enfermaria & 31 & 39,7 \\
\hline Centro de terapia intensiva & 7 & 9 \\
\hline Centro cirúrgico & 4 & 5,1 \\
\hline \multicolumn{3}{|l|}{ Motivos de retirada } \\
\hline Sem complicação documentada & 24 & 30,8 \\
\hline Suspeita de ICSR-CVCIV & 19 & 24,4 \\
\hline $\begin{array}{l}\text { Febre persistente sem foco } \\
\text { infeccioso }\end{array}$ & 10 & 12,8 \\
\hline Episódio de bacteremia e febre & 5 & 6,4 \\
\hline Infecção do sítio de saída & 5 & 6,4 \\
\hline Óbito & 5 & 6,4 \\
\hline Mau posicionamento & 4 & 5,1 \\
\hline Obstrução & 4 & 5,1 \\
\hline Tunelite & 2 & 2,6 \\
\hline
\end{tabular}

${ }^{\mathrm{I}}$ Hemotórax, problemas com o fio guia, necessidade de infusão de hemocomponentes e hematoma cervical; ${ }^{\text {II }} \mathrm{DP}$ : Desvio-padrão; ${ }^{\mathrm{III}}$ Médico contratado e médico intensivista; ${ }^{\text {IV }}$ ICSR-CVC: Infecção de corrente sanguínea relacionada ao cateter venoso central
Tabela 3. Relação de microrganismos isolados em hemoculturas e pontas de cateteres retirados por suspeita de infecção de corrente sanguínea relacionada ao cateter venoso central $(N=19)$, Ribeirão Preto-SP, 2006-2010

\begin{tabular}{|c|c|c|c|}
\hline \multirow[b]{2}{*}{ Hemocultura } & \multicolumn{3}{|c|}{ Ponta de cateter } \\
\hline & $\begin{array}{l}\text { Positivo } \\
\text { (n) }\end{array}$ & $\begin{array}{c}\text { Negativo } \\
\text { (n) }\end{array}$ & $\begin{array}{l}\text { ND } \\
\text { (n) }\end{array}$ \\
\hline S. epidermidis & - & 1 & 3 \\
\hline K. pneumoniae & 3 & - & - \\
\hline A. baumanni & 2 & - & - \\
\hline A. Iwoffii & 1 & - & - \\
\hline $\begin{array}{l}\text { A. baumannii + } \\
\text { S. epidermidis }\end{array}$ & 1 & - & - \\
\hline $\begin{array}{l}\text { A. baumannii + } \\
\text { S. epidermidis }\end{array}$ & $1^{*}$ & - & - \\
\hline S. maltophilia & - & - & 1 \\
\hline $\begin{array}{l}\text { S. maltophilia + } \\
\text { A. baumannii }\end{array}$ & - & - & 1 \\
\hline S. warneri & - & - & 1 \\
\hline S. aureus & - & - & 1 \\
\hline E. coli & 1 & - & - \\
\hline E. cloacae & - & - & 1 \\
\hline $\begin{array}{l}\text { Bacilo gram- } \\
\text {-negativo }\end{array}$ & - & - & 1 \\
\hline
\end{tabular}

ND: nấo documentado; * Embora na hemocultura tenha sido identificado o crescimento de duas espécies de microrganismos, na cultura da ponta do cateter houve crescimento apenas do $S$. epidermidis.

S. epidermidis: Staphylococcus epidermidis; K. pneumoniae: Klebsiella pneumoniae; A. baumanni: Acinetobacter baumanni; A. iwoffii: Acinetobacter lwoffi; S. maltophilia: Stenotrophomonas maltophilia; S. warneri: Staphylococcus warneri; S. aureus: Staphylococcus aureus; E. coli: Escherichia coli; E. cloacae: Enterobacter cloacae

\section{DISCUSSÃO}

A LMA representa a indicação mais comum de TCTH alogênico em adultos ${ }^{11}$, assim como mostram os dados evidenciados nessa investigação. O bussulfano, utilizado em grande parte dos regimes de condicionamento do presente estudo, está associado à dermatite, eritema e fragilidade cutânea por se tratar de um agente dermatologicamente tóxico, o que pode comprometer a cicatrização do local do implante do cateter ${ }^{12}$. Por esse motivo, a administração dos quimioterápicos de sete a dez dias após o implante do cateter minimiza os efeitos sobre a cicatrização ${ }^{13}$.

No entanto, os resultados deste estudo evidenciaram mediana de três dias, com três casos nos quais o regime se iniciou no mesmo dia do implante do cateter. Esse evento associado às condiçóes clínicas do paciente, como a função e número inadequados de plaquetas em decorrência da patologia de base e à quimioterapia com agente inibidor de fibroblasto, o tempo de cicatrizaçáo local pode se 
prolongar, assim como o tempo de exsudação da ferida, predispondo o local à infecção ${ }^{14}$.

O tempo de permanência médio do cateter in situ foi de 41 dias e o máximo de 118 dias. Em estudo realizado com pacientes submetidos ao TCTH autólogo, a mediana de permanência do cateter foi de 68 dias, variando de sete a 393 dias, indicando um período maior. No entanto, os autores relataram ampla variação ao revisar a literatura em relação à permanência do cateter de Hickman, chegando até 688 dias (medianas de 40 a 120 dias e tempo máximo de permanência de 232 a 688 dias) ${ }^{15}$.

Um aspecto que poderia justificar essa curta permanência na clientela estudada se relacionada ao fato de a equipe médica indicar a retirada do dispositivo, mesmo sendo de longa permanência, assim que o paciente não estiver mais o utilizando, com o intuito de prevenir possíveis complicaçóes tardias.

A infecção, assim como evidenciado no presente estudo, é a complicação mais comum no uso do cateter venoso central de longa permanência ${ }^{16}$, sendo a ICSRCVC caracterizada como risco intermediário entre os cateteres tunelizados e implantados cirurgicamente $(1,6$ por 1000 cateteres/dia) $)^{4}$.

Os pacientes que evoluíram para neutropenia severa por período superior a dez dias representaram a maioria dos casos $(94,3 \%)$, sendo que, entre outros fatores de risco, a imunossupressão apresentada pelo paciente submetido ao TCTH alogênico se constitui em um dos mais importantes para o desenvolvimento de ICSR-CVC. Ainda, uma vez instituída a terapia antimicrobiana, sua duração e sucesso relacionam-se intimamente com a contagem de neutrófilos ${ }^{4}$.

Outros aspectos presentes nos pacientes submetidos ao TCTH que podem se constituir em fatores de risco para o desenvolvimento de infecçôes são o tratamento com altas doses de quimioterápicos, o frequente manuseio do dispositivo ${ }^{17}$ e a quebra de barreiras cutâneas e mucosas.

Ressalta-se que, dos 36 pacientes que receberam NPT, na maioria das vezes em função do desenvolvimento de mucosite, $44,4 \%$ tiveram alguma infecçáo relacionada ao cateter. Nesse sentido, a literatura aponta relação importante da ocorrência de infecçôes de cateter com o uso de acessos venosos semi-implantados para administração de NPT?

Com o avanço tecnológico relacionado ao desenvolvimento dos CVC, as espécies de microrganismos causadores de infecçóes relacionadas ao cateter têm mudado nas últimas décadas ${ }^{4}$. Assim como identificado neste estudo, pesquisadores ${ }^{18-19}$ evidenciaram que as bactérias gram-negativas representam os principais microrganismos causadores de infecção, sendo Klebsiella pneumoniae e Acinetobacter baumanni os mais frequentes identificados em uma unidade de cuidados intensivos em neonatos ${ }^{18-19}$. A imunossupressão que ocorre nos pacientes submetidos ao TCTH pode revelar relação com o quadro apresentado nesses estudos, realizados com uma população que possui o sistema imunológico ainda em amadurecimento.

A Klebsiella pneumoniae, isolada em $30 \%$ dos casos, trata-se de importante patógeno oportunista, especialmente em pacientes imunocomprometidos. Acredita-se que, pela sua capacidade de formação de biofilme em dispositivos como cateteres, apresentam importante papel no desenvolvimento de infeçóes nosocomiais ${ }^{20}$. O Acinetobacter baumanni, isolado com a mesma frequência, é uma bactéria oportunista, frequentemente envolvida em surtos de infecção, capaz de contaminar e persistir em várias superfícies, disseminando-se entre os pacientes e em diversos contextos ${ }^{21}$.

Para realização de um diagnóstico definitivo de ICSR-CVC, alguns critérios podem ser utilizados, sendo um deles a necessidade de crescimento do mesmo microrganismo em pelo menos uma amostra de sangue periférico e na cultura de ponta do cateter ${ }^{22}$. Entre as suspeitas de ICSR-CVC deste estudo, a confirmação ocorreu em 47,4\% dos casos ( $\mathrm{n}=9$ ). Em nove situações, não foi possível identificar o resultado da cultura da ponta do cateter no prontuário do paciente; e, em apenas um caso, essa cultura foi negativa.

Assim como na presente investigaçáo, em um recente estudo com pacientes que realizaram o TCTH, a suspeita ou confirmação de infecçôes representam o principal motivo de retirada de cateteres venosos centrais ${ }^{23}$. Embora a remoção precoce do cateter seja indispensável em alguns casos, esse ato prematuro ou desnecessário pode resultar em interrupção ou atraso do tratamento, aumento do desconforto do paciente, ansiedade, custos elevados e aumento do tempo de hospitalizaçáo ${ }^{4}$.

Tendo em vista que as infecçôes de corrente sanguínea podem ser amplamente preveníveis ${ }^{4}$, cuidados visando à minimização dessa complicação devem ser tomados. Para que se obtenha sucesso na inserçáo do cateter, a experiência e habilidade do profissional que realiza o procedimento são fundamentais ${ }^{4,8}$. Além disso, o ensino de todos os profissionais que manuseiam o dispositivo e a educação individualizada e supervisionada de pacientes e cuidadores são considerados medidas efetivas, acessíveis e que diminuem as taxas de infecçãón.

\section{CONCLUSÃO}

Diante dos dados apresentados, infere-se que o intervalo de tempo entre o implante cirúrgico do cateter de Hickman e o início do regime de condicionamento, menor que o preconizado pela literatura, pode ter prolongado o período de cicatrização e a presença de exsudato, predispondo o paciente a infecçóes, que foram as principais responsáveis pela curta permanência do cateter. 
Os microrganismos Klebsiella pneumoniae e Acinetobacter baumanni foram isolados com maior frequência nas hemoculturas.

A partir da análise dos resultados, é possível compreender os motivos de retirada do cateter de Hickman, o que auxiliará o enfermeiro a propor intervençóes para minimizar complicaçôes advindas desse dispositivo intravascular, de forma a corroborar sua permanência por um período mais prolongado, em coerência com a proposta de ser um dispositivo de longa duração seguro.

Nesse sentido, considerando que o principal motivo de retirada documentado foi a suspeita de infecção ou a sua confirmação, medidas visando à redução dessa complicação foram iniciadas, entre elas: atualização da equipe responsável pelo manuseio desse dispositivo, discussão com a equipe interdisciplinar sobre a possibilidade de aguardar o tempo mínimo preconizado entre o implante do cateter e o início do regime de condicionamento e o desenvolvimento atual de uma investigação acerca do uso de uma cobertura impregnada com antisséptico no sítio de saída do cateter.

$\mathrm{O}$ presente estudo apresenta limites no que tange à dificuldade na obtenção de dados consistentes nos registros dos prontuários. Enfatiza-se a necessidade de ensaios clínicos controlados e aleatorizados para um mapeamento mais profundo sobre os diversos fatores envolvidos na permanência do cateter de Hickman.

\section{CONTRIBUIÇÕES}

Juliane Zagatti Alves Pereira participou da concepção e planejamento do estudo; obtençáo, análise e interpretação dos dados; redação e aprovação da versão final do artigo. Fernanda Titareli Merizio Martins Braga, Lívia Maria Garbin, Lais Carvalho Castanho participaram do planejamento do estudo; análise e interpretação dos dados; redação, revisão crítica e aprovação da versão final do artigo. Renata Cristina de Campos Pereira Silveira participou da concepção e planejamento do estudo; análise e interpretação dos dados; revisão crítica e aprovação da versão final do artigo.

\section{Declaraçáo de Conflito de Interesses: Nada a Declarar.}

\section{REFERÊNCIAS}

1. Li HW, Sykes M. Emerging concepts in haematopoietic cell transplantation. Nat Rev Immunol. 2012; 12(6):403-16.

2. Bishop MR. Principles of hematopoietic stem cell transplantation to treat hematologic malignances. In: Sekeres MA, Kalaycio ME, Bolwell BJ, editors. Clinical malignant hematology. New York: McGraw-Hill; 2007. p. 975-84.
3. Cunha MALC, Leite JL. O ser portador de um cateter venoso central: a percepção do cliente e a contribuição para a enfermagem. Rev Bras Cancerol. 2008; 54(2):139-45.

4. Schiffer CA, Mangu PB, Wade JC, Camp-Sorrell D, Cope DG, El-Rayes BF, et al. Central venous catheter care for the patient with cancer: american society of clinical oncology clinical practice guideline. J Clin Oncol. 2013; 31(10):1357-70. Epub 2013 Mar 4.

5. Poutsiaka DD, Price LL, Ucuzian A, Chan GW, Miller KB, Snydman DR. Blood stream infection after hematopoetic stem cell transplantation is associated with increased mortality. Bone Marrow Transplant. 2007; 40(1):63-70. Epub 2007 Apr 30.

6. Yazbek G, Zerati AE, Langer M, Malavolta LC, Nishinari K, Wolosker N. Utilização dos cateteres de Hickman em transplante autólogo de medula óssea: análise de 49 cateteres implantados. Acta Oncol Bras. 2002; 22(3): 299-305.

7. Liu CY, Lai YC, Huang LJ, Yang YW, Chen TL, Hsiao LT, et al. Impact of bloodstream infections on outcome and the influence of prophylactic oral antibiotic regimens in allogeneic hematopoietic SCT recipients. Bone Marrow Transplant, 2011; 46(9):1231-9. Epub 2010 Nov 29.

8. Albuquerque MP. Cirurgia dos cateteres de longa permanência (CLP) nos centros de transplante de medula óssea. Medicina (Ribeirão Preto) 2005; 38(2):125-42.

9. Nieboer P, de Vries EG, Mulder NH, Rodenhuis S, Bontenbal M, Van der Wall E, et al. Factors influencing catheter-related infections in the Dutch multicenter study on high-dose chemotherapy followed by peripheral SCT in high-risk breast cancer patients. Bone Marrow Transplant. 2008; 42(7):475-81. Epub 2008 Jul 14.

10. O'Grady NP, Alexander M, Burns LA, Dellinger EP, Garland J, Heard SO, et al. Guidelines for the prevention of intravascular catheter-related infections. Clin Infect Dis. 2011; 52(9):162-93.

11. Passweg JR, Baldomero H, Bregni M, Cesaro S, Dreger P, Duarte RF, et al. Hematopoietic SCT in Europe: data and trends in 2011. Bone Marrow Transplant 2013; 48(9):1161-7. Epub 2013 Apr 15.

12. Benhamou E, Fessard E, Com-Nougué C, Beaussier PS, Nitenberg G, Tancrède $C$, et al. Less frequent catheter dressingchanges decrease local cutaneous toxicity of highdose chemotherapy in children, without increasing the rate of catheter-related infections: results of a randomized trial. Bone Marrow Transplant. 2002; 29(8):653-8.

13. Payne WG, Naidu DK, Wheeler CK, Barkoe D, Mentis $\mathrm{M}$, Salas RE, et al. Wound healing in patients with cancer. Eplasty 2008; 8:68-90.

14. Silveira RCCP, Braga FTMM, Garbin LM, Galvão CM. The use of polyurethane transparent film in indwelling central venous catheter. Rev Lat Am Enferm. 2010; 18(6):1212-20. 
15. Almeida MH. Factibilidade do uso do cateter tipo Hickman em uma enfermaria geral de hematologia [dissertação]. Campinas: Universidade Estadual de Campinas; 2005.

16. Park KH, Cho OH, Lee SO, Choi SH, Kim YS, Woo JH, et al. Development of subsequent bloodstream infection in patients with positive Hickman catheter blood cultures and negative peripheral blood cultures. Diagn Microbiol Infect Dis. 2011; 70(1):31-6. Epub 2011 Mar 12.

17. Kim DH, Bae NY, Sung WJ, Kim JG, Kim SW, Sohn SK, et al. Hickman catheter site infections after allogeneic stem cell transplantation: a single-center experience. Transplant Proc. 2004 Dec; 36(10):3203-7.

18. Fahmey SS. Early-onset sepsis in a neonatal intensive care unit in Beni Suef, Egypt: bacterial isolates and antibiotic resistance pattern. Korean J Pediatr. 2013; 56(8):332-7. Epub 2013 Aug 27.

19. Mai JY, Dong L, Lin ZL, Chen SQ. [Investigation and analysis of nosocomial infection in neonates]. Zhonghua Er Ke Za Zhi. 2011; 49(12):915-20. Chinês
20. Schroll C, Barken KB, Krogfelt KA, Struve C. Role of type 1 and type 3 fimbriae in Klebsiella pneumoniae biofilm formation. BMC Microbiol. 2010; 10:179.

21. Romanelli RMC, Jesus LA, Clemente WT, Lima SSS, Rezende EM, Coutinho RL, et al. Outbreak of resistant Acinetobacter baumannii- measures and proposal for prevention and control. Braz J Infect Dis. 2009; 13(5):341-7.

22. Mermel LA, Allon M, Bouza E, Craven DE, Flynn P, O'Grady NP, et al. Clinical Practice Guidelines for the Diagnosis and Management of Intravascular Catheter-Related Infection: 2009 Update by the Infectious Diseases Society of America. Clin Infect Dis. 2009;49(1):1-45. Erratum in: Clin Infect Dis. 2010 Apr 1; 50(7):1079. Dosage error in article text. ; Clin Infect Dis. 2010 Feb 1; 50(3):457.

23. Castanho LC, Silveira RCCP, Braga FTMM, Canini SRMS, Reis PED, Voltarelli JC. Motivo de retirada do cateter de Hickman em pacientes submetidos ao transplante de células-tronco hematopoéticas. Acta Paul Enf 2011; 24(2):244-8. 


\begin{abstract}
Introduction: The use of the Hickman's catheter is critical to enable complex therapies. Nevertheless, its safe stay in situ requires knowledge of the professionals who handle this device about the major complications that lead to its withdrawal. Objective: Analyze the permanence of the Hickman catheter in patients undergoing allogeneic hematopoietic stem cell transplantation. Method: A descriptive cross-sectional study with retrospective data collection. Seventy-three medical records of patients were identified in the interest period of the study, of which three were excluded for having incomplete information. Data were analyzed by using descriptive statistics. Results: 78 catheters were implanted in 70 patients. The mean length of stay of the devices was 41 days $(\mathrm{sd}=26.78)$. The average time between catheter insertion and start of the conditioning regimen was four days ( $\mathrm{s} d=4.6)$. Regarding the indication for catheter removal, in $26(33.4 \%)$ cases, the withdrawal was indicated due to suspicion of catheter-related infections. In other $24(30.8 \%)$ cases, indicating occurred after the patient has initiated outpatient follow-up, however, without complications documented in the patient record. Conclusion: The Hickman catheter usually cause complications, among them, the infection which leads to a short length of stay of the device in situ, contradicting its proposed for long time, and often be removed without explicit criterion record before the end of therapy. This research contributes to nurses of clinical practice to identify the main reasons for catheter removal and provide data that can support proposals for interventions to minimize them.

Key words: Catheterization, Central Venous-nursing; Nursing Care; Bone Marrow Transplantation-nursing; CatheterRelated Infections-nursing; Hematopoietic Stem Cell Transplantation-nursing
\end{abstract}

\title{
Resumen
}

Introducción: El uso del catéter de Hickman es fundamental para permitir terapias complejas, sin embargo, su segura permanencia requiere el conocimiento de los profesionales que lo manipulan, sobre todo en las principales complicaciones que conducen a su retirada. Objetivo: Analizar la permanencia del catéter de Hickman en pacientes sometidos a trasplante alogénico de células madre hematopoyéticas. Método: Un estudio de corte transversal con recopilación de datos retrospectiva. Se identificaron 73 registros médicos de pacientes en el período de estudio, de los cuales, tres fueron excluidos pues tenían una información incompleta. Los datos fueron analizados utilizando estadística descriptiva. Resultados: Fueron implantados 78 catéteres en 70 pacientes, con tiempo de permanencia media de 41 días ( $\mathrm{sd}=26.78$ ). El tiempo promedio transcurrido entre la inserción del catéter y el inicio del régimen de acondicionamiento fue de cuatro días $(\mathrm{sd}=4.6)$. En 26 casos $(33.4 \%)$, la indicación de extracción del catéter fue por sospecha de infecciones y en otros 24 casos (30.8\%), la extracción ocurrió después del seguimiento ambulatorio, pero sin complicaciones documentadas en el historial médico. Conclusión: El catéter de Hickman generalmente causa complicaciones, entre ellas, la infección, lo que conduce a un corto tiempo de permanencia del dispositivo in situ, contrariando su propuesta de larga permanencia, además de que, a menudo, es retirado sin registro del criterio explícito antes del final de la terapia. Esta investigación contribuye a la práctica clínica del enfermero para identificar las principales razones de la extracción del catéter y proporcionar datos que puedan apoyar las propuestas de intervenciones para minimizarlos.

Palabras clave: Cateterismo Venoso Central-enfermería; Atención de Enfermaría; Trasplante de Medula Óseaenfermería; Infecciones Relacionadas con Catéteres-enfermería; Trasplante de Células Madre Hematopoyéticasenfermería 